Jane Hodgkinson, John Saffell, Jonathan Luff, John Shaw, Jeremy Ramsden, Carlos Huggins, Robert Bogue and Roger Carline

\title{
Gas sensors 2. The markets and challenges
}

ABSTRACT. This paper looks at the market for gas and vapour sensors and its drivers, including legislative (regulatory) ones. The nature of the market is reviewed, considering market pull and technology push. The various market sectors, including medical and food, security, indoor and outdoor air quality (both domestic and industrial) and industrial process control are analysed in detail. Societal needs are assessed, and scientific and technological challenges for the future are discussed. The paper ends with a summary of priorities.

Nanotechnology Perceptions 5 (2009) 83-107

Nonsubscribers: purchase individual article 